\section{Intrahepatic steal after Fontan operation with partial hepatic exclusion}

Ivatury M. Rao, MD, Jeffrey S. Swanson, MD, Hagop Hovaguimian, MD, David M. McIrvin, MD, Douglas $\mathrm{H}$. King, MD, Anthony P. Furnary, MD, Joanie Livermore, CMI, and Albert Starr, MD, Portland, Ore.

We would like to share our experience with a patient in whom a progressive right-to-left shunt developed because of intrahepatic steal.

From the Children's Cardiac Center of Oregon, P.C., 9155 S.W. Barnes Road, Suite 240, Portland, OR 97225-6689.

J THOraC Cardiovasc Surg 1995;109:180-1

Copyright (C 1995 by Mosby-Year Book, Inc. $0022-5223 / 95 \$ 3.00+0 \quad \mathbf{1 2 / 8 / 5 5 9 9 6}$

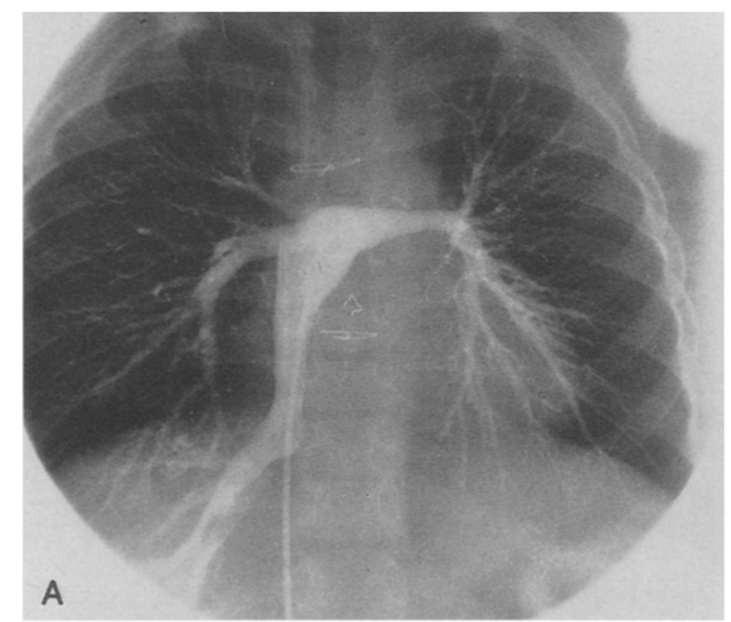

B

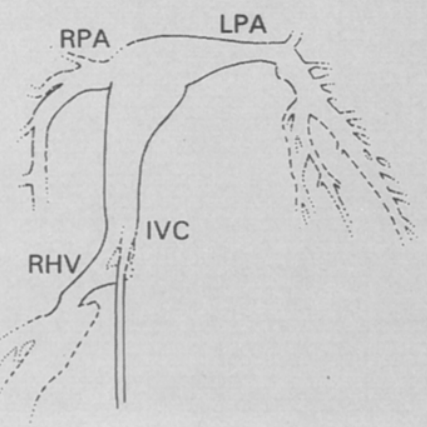

Fig. 1. $R P A$, Right pulmonary artery; $L P A$, left pulmonary artery; $I V C$, inferior vena cava; $R H V$, right hepatic vein.
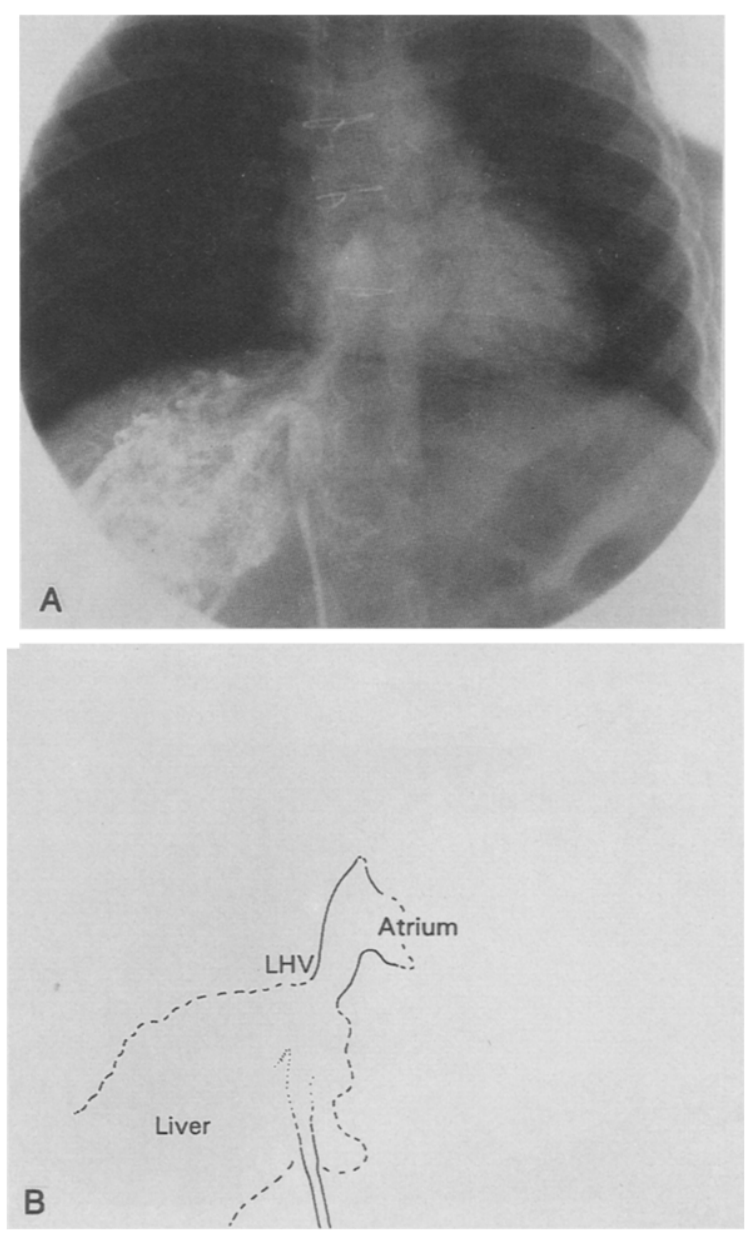

Fig. 2. $L H V$, Left hepatic vein.

A $31 / 2$-year-old child with hypoplastic left heart syndrome (HLHS) had undergone the Norwood stage I procedure as a neonate and a stage II (bidirectional Glenn shunt) several months later. At the age of 2 years she was treated with the complete Fontan operation (Norwood stage III), lateral tunnel, and hepatic vein exclusion by partition of the inferior vena cava (IVC). The left hepatic vein had been excluded from the tunnel and was draining to the common atrium. The right hepatic vein drained into the IVC. The child progressed well and was discharged without overt cyanosis or evidence of a right-toleft shunt.

All of her previous treatment was done at another institution, and she was brought to our center for followup. Over a period of 8 months increasing cyanosis and polycythemia developed, and the patient underwent cardiac catheterization. The resting arterial saturation was in the low seventies and fell further upon exertion. Cardiac catheterization revealed no baffle leaks. The right hepatic vein was in continuity with the IVC and the lateral tunnel. Contrast medium filled the pulmonary arteries from the IVC (Fig. 1). A slightly delayed view showed that contrast medium also flowed retrogradely from the IVC into the 


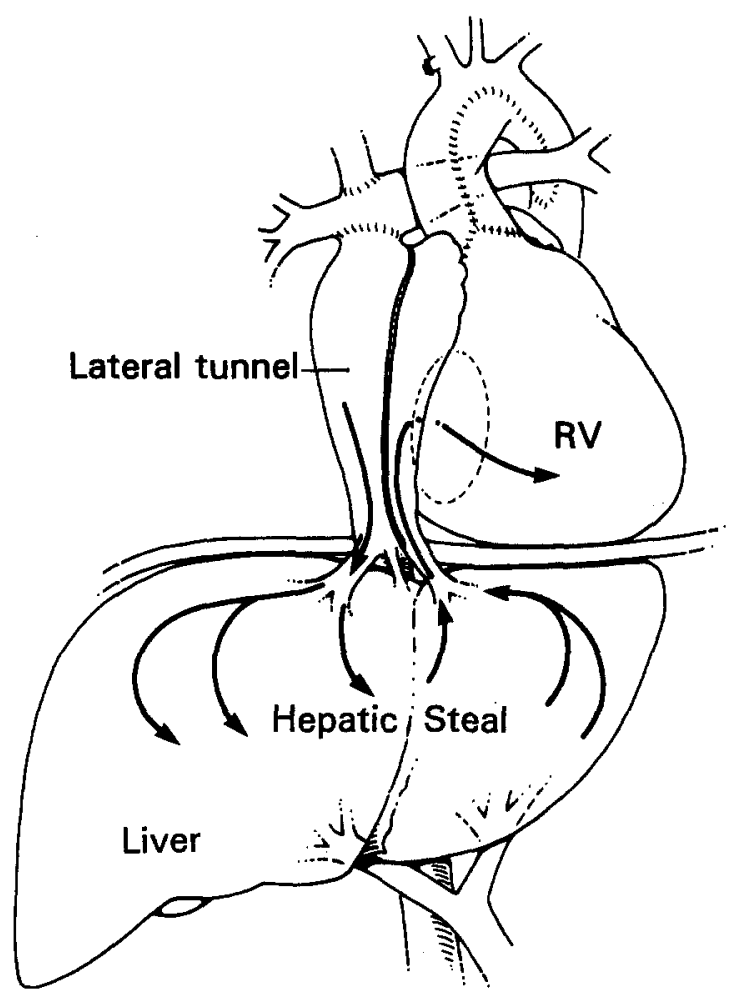

Fig. 3. $R V$, Right ventricle.

right hepatic vein and then into the hepatic tissue. It was noticed that after the contrast medium filled the hepatic sinusoidal bed, it coalesced to fill the left hepatic vein and then the common atrium, thus showing a steal phenomenon through the liver (Fig. 2). This hepatic steal created a right-to-left shunt because of the hepatic vein exclusion that had been done with the Norwood stage III operation. The relatively lower pressure in the left hepatic vein and atrium was "stealing" from the Fontan circulation, which accounted for the increasing cyanosis. Presumably, the interconnecting hepatic venous network allowed this to occur. In addition to this intrahepatic right-to-left shunt, the effective pulmonary blood flow also was reduced by this steal (Fig. 3).

This child underwent reoperation. A triangular piece of the existing polytetrafluoroethylene material was excised at the lower end of the lateral tunnel, and a new baffle was constructed to divert the left hepatic venous drainage into the lateral tunnel. After the reoperation the child's oxygen saturation was normal.

In an attempt to reduce effusions, liver congestion, and other adverse effects of the Fontan operation, the Philadelphia group ${ }^{1}$ introduced partial hepatic vein exclusion by partitioning the IVC. Although this modification is attractive for its lessening of the morbidity of the Fontan operation, due consideration should be given to the possibility that late hepatic steal may occur. Our patient raises the spectre of late malfunction of the Fontan circulation after hepatic vein exclusion.

\section{REFERENCE}

1. Jacobs ML, Norwood WT. Fontan operation: influence of modifications on morbidity and mortality. In: Abstracts, Thirtieth Anniversary Meeting. The Society of Thoracic Surgeons, 1993-1994:44.

\section{Primary cutaneous Nocardia farcinica infection after heart transplantation: A case report}

\author{
Wolfgang Rees, MD, Stephan Schüler, MD, \\ Manfred Hummel, MD, Susanne Hempel, MD, \\ Jörg Möller, MD, and Roland Hetzer, MD, \\ Berlin, Germany
}

Nocardia is known to cause severe infection, especially in immunocompromized patients with malignant disease, viral immunodeficiency syndrome, or in those patients receiving immunosuppressive therapy. ${ }^{1}$ To our knowledge, a total of 47 cases of nocardiosis in cardiac transplant recipients have been published, ${ }^{2}$ but this is the first case of primary cutaneous infection caused by Nocardia farcinica in a transplant recipient, without dissemination of the infection and without pulmonary or cerebral affection.

In May 1992, a 57-year-old male patient underwent orthotopic cardiac transplantation because of end-stage ischemic heart disease. Revascularization operations had already been performed in 1977 and 1988. The treatment regimen consisted of triple drug immunosuppression with initial induction therapy with rabbit antithymocyte globulin $100 \mathrm{mg}$ for 3 days. The intraoperative and perioperative course was uneventful, and the rehabilitation program was performed as usual. No rejection episodes had to be controlled.

Seven weeks after transplantation, the patient began having a backache. Computed tomography (CT) of the spine showed typical signs of severe osteoporosis and osteoporotic fractures of the eighth and eleventh thoracic vertebrae. Treatment was started with conservative drug therapy and a corset. One month later at home, low back pain increased again and an emergency physician started analgesic treatment by intramuscular injection of nonsteroidal antiphlogistics. Radiologic reinvestigation showed beginning osteoporotic fracture of the tenth thoracic vertebra. Three weeks later, swelling, tenderness, and increasing pain developed in the left groin and thigh, and the man was admitted to his local hospital because of high fever, chills, malaise, and a swollen left thigh. A CT scan revealed a circumscript hypodense intramuscular mass medial above the left knee, reaching the left groin (Fig. 1). Physical examination on admission was unremarkable

From the German Heart Institute Berlin, Department of Thoracic and Cardiovascular Surgery, Berlin, Germany.

J Thorac CARdiovasc Surg 1995;109:181-3

Copyright $\subseteq 1995$ by Mosby-Year Book, Inc.

$0022-5223 / 95 \$ 3.00+0 \quad \mathbf{1 2} / \mathbf{8} / \mathbf{5 2 6 2 2}$ 